\title{
On-demand versus continuous rocuronium infusion for deep neuromuscular relaxation in patients undergoing thoraco- laparoscopic esophagectomy: a randomized-controlled clinical trial (DEPTH)
}

\section{Perfusion continue versus bolus intermittent sur demande de rocuronium pour procurer une relaxation neuromusculaire profonde aux patients subissant une osophagectomie par thoraco-laparoscopie : une étude clinique randomisée contrôlée (DEPTH)}

\author{
Denise P. Veelo, MD, PhD • Suzanne S. Gisbertz, MD, PhD • Jan M. Binnekade, MsC, PhD • \\ Rebekka A. Hannivoort, MD · Johanneke A. Bosman, MD • Bart F. Geerts, MD, PhD (D) • \\ Manfred Blobner, MD, PhD • Mark I. van Berge Henegouwen, MD, PhD • \\ Markus W. Hollmann, MD, PhD
}

Received: 16 October 2018/Revised: 28 February 2019/Accepted: 2 March 2019/Published online: 25 April 2019

(C) The Author(s) 2019

\begin{abstract}
Purpose Deep neuromuscular blockade (NMB) can improve surgical conditions and possibly pain after lowrisk laparoscopic surgery. We hypothesized that targeting a deep level of NMB by a continuous compared with an on-
\end{abstract}

Electronic supplementary material The online version of this article (https://doi.org/10.1007/s12630-019-01373-0) contains supplementary material, which is available to authorized users.

D. P. Veelo, MD, $\mathrm{PhD}$ - R. A. Hannivoort, MD .

J. A. Bosman, MD - B. F. Geerts, MD, PhD ( $)$.

M. W. Hollmann, MD, PhD

Department of Anesthesiology, Academic Medical Center,

Meibergdreef 9, H1-158, 1105 AZ Amsterdam, The Netherlands

e-mail: B.F.Geerts@amc.uva.nl

S. S. Gisbertz, MD, PhD - M. I. van Berge Henegouwen, MD, $\mathrm{PhD}$

Department of Surgery, Academic Medical Center, Amsterdam,

The Netherlands

J. M. Binnekade, $\mathrm{MsC}, \mathrm{PhD}$

Department of Intensive Care Medicine, Academic Medical

Center, Amsterdam, The Netherlands

M. Blobner, $\mathrm{MD}, \mathrm{PhD}$

Klinik für Anästhesiologie der Technischen Universität

München, Munich, Germany demand infusion of rocuronium could improve surgical conditions in patients undergoing thoraco-laparoscopic esophagectomy.

Methods In this single-centre, randomized-controlled, double-blind trial, patients received either a continuous infusion of rocuronium $0.6 \mathrm{mg} \cdot \mathrm{kg}^{-1} \cdot \mathrm{hr}^{-1}$ (intervention) or $\mathrm{NaCl} 0.9 \%$ (control). Both surgeon and anesthesiologist were blinded to group assignment and the train-of-four measurements. Open-label rocuronium was given if requested (i.e., on-demand) by the surgeon. At the end of surgery, sugammadex was given if necessary to reverse the NMB. The primary outcome was the quality of surgical conditions during the abdominal phase of the operation as measured by the surgical rating scale (SRS). Secondary outcomes included the thoracic SRS, number of on-demand boluses, intraoperative surgical events, pain scores (up to $12 \mathrm{hr}$ postoperatively), and duration of surgery.

Results The median [interquartile range] abdominal SRS was not different between the intervention (4 [4-5]) and control (4 [4-5]) groups (median difference, 0; $95 \%$ confidence interval, 0 to $0 ; P=0.45)$. The thoracic $S R S$ was 4 [4-4] in both groups $(P=0.23)$. The median number of rocuronium bolus requests was higher in the control group compared with the intervention group (3 [3-6] vs 1 [0-2], respectively; $P<0.01)$. There were no between-group 
differences in intraoperative surgical events $(P=0.05)$, pain scores (overall $P>0.05)$, or duration of surgery $(P=0.95)$. Conclusions Continuous rocuronium infusion did not improve surgical conditions when boluses of rocuronium were available on-demand. No major benefits in other outcomes were seen.

Trial registration EUDRACT (2014-002147-18); registered 19 May, 2014 and clinicaltrials.gov (NCT02320734); registered 18 December, 2014.

\section{Résumé}

Objectif Les blocs neuromusculaires (BNM) profonds peuvent améliorer les conditions chirurgicales et possiblement atténuer la douleur après une chirurgie par laparoscopie à faible risque. Nous avons émis l'hypothèse qu'en ciblant un niveau profond de BNM à l'aide d'une perfusion continue par rapport à $d u$ rocuronium sur demande, les conditions chirurgicales des patients subissant une oesophagectomie par thoraco-laparoscopie pourraient être améliorées.

Méthode Dans cette étude randomisée contrôlée à double insu et mono-site, les patients ont reçu une perfusion continue de rocuronium $0,6 \mathrm{mg} \cdot \mathrm{kg}^{-1} \cdot \mathrm{h}^{-1}$ (groupe intervention) ou une solution de $\mathrm{NaCl} \mathrm{0,9 \%} \mathrm{(témoin).} \mathrm{Le}$ chirurgien et l'anesthésiologiste étaient tous deux ignorants de l'attribution de groupe et des mesures de la curarisation par neurostimulateur. Du rocuronium a été donné en plus si demandé par le chirurgien. À la fin de la chirurgie, du sugammadex a été administré au besoin pour neutraliser le BNM. Le critère d'évaluation principal était la qualité des conditions chirurgicales pendant la phase abdominale de l'opération telle que mesurée par l'échelle d'évaluation de la chirurgie (Surgical rating scale-EEC). Les critères secondaires étaient l'EEC thoracique, le nombre de bolus demandés, les événements chirurgicaux peropératoires, les scores de douleur (jusqu'à $12 \mathrm{~h}$ postopératoires) et la durée de la chirurgie.

Résultats L'EEC abdominale médiane [écart interquartile] n'était pas différente entre les groupes intervention (4 [4-5]) et témoin (4 [4-5]) (différence médiane, 0; intervalle de confiance $95 \%, 0$ à $0 ; P=0,45)$. L'EEC thoracique était de 4 [4-4] dans les deux groupes $(P=0,23)$. Le nombre moyen de bolus de rocuronium demandés était plus élevé dans le groupe témoin par rapport au groupe intervention (3 [3-6] vs 1 [0-2], respectivement; $P<0,01)$. Il n'y avait pas de différences entre les groupes en matière d'événements chirurgicaux peropératoires $(P=0,05)$, de scores de douleur (globalement $P>0,05)$, ou de durée de la chirurgie $(P=0,95)$.

Conclusion Une perfusion continue de rocuronium n'a pas amélioré les conditions chirurgicales lorsque les bolus de rocuronium étaient disponibles sur demande. Aucun autre bénéfice majeur n'a été observé selon nos autres critères d'évaluation.
Enregistrement de l'étude EUDRACT(2014-002147-18); enregistrée le 19 mai 2014 et clinicaltrials.gov (NCT02320734); enregistrée le 18 décembre 2014.

Providing deep neuromuscular blockade (NMB) for laparoscopic surgery allows for lower inflation pressures compared with minimal or moderate NMB; it can also improve the ease and duration of surgery. This may result in a reduction in pain by means of decreased tissue damage and activation of peritoneal nociceptors and ultimately, a decrease in morbidity. ${ }^{1-8}$ Nevertheless, the risk of intraoperative awareness, prolonged emergence from anesthesia, and concerns regarding residual NMB may limit widespread implementation of deeper levels of NMB. ${ }^{9}$ In addition, recent evidence suggests that the indiscriminate use of neostigmine after deep NMB may increase the risk of postoperative respiratory complications. ${ }^{10,11}$ Conversely, sugammadex provides a rapid and dose-dependent reversal of NMB with a minimal side-effect profile. $^{12}$ Nevertheless, the costs associated with the use of high dose sugammadex to reverse very deep levels of NMB are considerable. They may be an important financial barrier to using deep NMB if improvement in quality of care or cost saving elsewhere cannot be shown. ${ }^{9}$

Most studies assessing the advantages of deep NMB have been done in patients undergoing low-risk abdominal procedures or bariatric surgery. ${ }^{1-8}$ Thoraco-laparoscopic esophageal surgery is generally a six- to eight-hour highrisk procedure encompassing laparoscopic (abdominal) and thoracic phases. ${ }^{13}$ The possible advantages of deep NMB for surgical conditions during thoracoscopy have not been studied thus far.

The primary objective of this study was to quantify the difference in effect of an on-demand only NMB regime $v s$ a continuous NMB infusion aiming for deep relaxation during the abdominal phase (i.e., in the anatomical region near the diaphragm) during thoraco-laparoscopic esophagectomy. The secondary objectives examined surgical conditions during the thoracic phase, the number of on-demand boluses, intraoperative adverse events, duration of surgery, and postoperative pain. We hypothesized that surgical conditions would be improved by a deeper level of NMB.

\section{Methods}

Study design and participants

We performed a single-centre, randomized-controlled, double-blind trial. The rationale and design of this study 
have been described in detail elsewhere. ${ }^{14}$ No changes in the design were made after the start of the trial, other than an adjustment in the sugammadex dose (as described earlier). ${ }^{14}$ All adults undergoing a thoraco-laparoscopic esophagectomy (i.e., McKeown or Ivor Lewis variant) at the Academic Medical Center, Amsterdam, The Netherlands were eligible for inclusion. The trial was performed between 1 February 2015 and 30 September 2016 and patient follow-up was at three months. Patients were informed about the study at our Department of Anesthesiology preoperative clinic and were contacted afterwards by the study investigator to determine their interest in participating. All patients gave written informed consent on the surgical ward the day before surgery. The exclusion criteria included age $<18 \mathrm{yr}$, pregnancy, known allergies to aminosteroid-type muscle relaxants or sugammadex, severe kidney dysfunction (glomerular filtration rate $<30 \mathrm{~mL} \cdot \mathrm{min}^{-1} \cdot 1.73 \mathrm{~m}^{-2}$ ) or dialysis, liver dysfunction, any neuromuscular diseases, carcinomatosis, as well as the use of anti-epileptic agents, lithium, or drugs containing kinine.

This investigator-initiated study was approved on 16 December, 2014 by the local ethics committee (Academic Medical Centre, registration number 2014_211\#B20141036) and registered at clinicaltrials.gov (NCT02320734). The study was conducted according to the principles of the Declaration of Helsinki (version of Fortaleza, Brazil 2013) and in accordance with the Medical Research Involving Human Subjects Act. ${ }^{15}$ A data monitoring safety board assessed the study. Reporting was done according to the CONSORT statement. ${ }^{16}$

\section{Randomization and blinding}

Before induction of anesthesia, the study investigator randomly allocated each patient (with random blocks of two, four, and six patients) to either the intervention or the control group (1:1) using a web-based randomization module, which additionally stratified the allocation according to the type of surgery (three-stage or two-stage procedure). Treatment allocation was blinded to those involved (i.e., patient, surgeon, and anesthesiologist). Double blinding was maintained during the study unless the anesthesiologist in charge needed to unblind allocation for clinical reasons (though no patients were unblinded). The coordinating investigator remained blinded until the study was completed and ready for analysis.

Study procedures

The study protocol has been previously described in detail. ${ }^{14}$ Briefly, after induction of anesthesia, a rocuronium infusion was started $\left(0.60 \mathrm{mg} \cdot \mathrm{kg}^{-1} \cdot \mathrm{hr}^{-1}\right)$ in patients of the intervention group and a saline infusion $\left(0.9 \% \mathrm{NaCl}, 0.06 \mathrm{~mL} \cdot \mathrm{kg}^{-1} \cdot \mathrm{hr}^{-1}\right)$ in patients of the control group. Extra doses of open-label rocuronium $0.30 \mathrm{mg} \cdot \mathrm{kg}^{-1}$ (i.e., on-demand) could be requested by either surgeon or anesthesiologist in both groups for the following indications: high peak inflation pressure $(>35 \mathrm{mmHg})$, high abdominal $(>15 \mathrm{mmHg})$ and/or thoracic $(>8 \mathrm{mmHg})$ insufflation pressures, as well as movement, breathing, or straining of the patient.

Surgical conditions were assessed using the five-point Leiden surgical rating scale (SRS) ${ }^{2}$ as well as a numeric rating scale. ${ }^{3}$ The SRS score comprised an ordinal scale from 1 (poor surgical conditions) to 5 (optimal surgical conditions). It was developed to specifically determine surgical conditions during various types of laparoscopic surgeries and has shown reproducibility and low interobserver variability (Table 1$)^{2,7}$ The numeric rating scale ranges from 1 (worst surgical conditions) to 100 (best surgical conditions). During the operation, the surgeons were asked to rate conditions by giving a number between $1-5$ and 1-100, respectively. Prior to the start of the study, both surgeons (S.G. and M.B.H.) were trained in using the SRS and numeric rating scale correlate. Consensus on the score had to be reached between surgeons. If consensus was not reached, the score of the senior surgeon was noted. Surgical conditions were assessed directly before and five minutes after any extra bolus of rocuronium following the surgeons' request (i.e., on-demand SRS observation).

\section{Neuromuscular blockade monitoring}

Neuromuscular blockade was monitored at the foot (thus blinding the anesthesiologist and the surgeon) using the flexor hallucis brevis muscle and the tibialis posterior nerve. The stimulating electrodes were placed next to the median malleolus with the measuring probe fixed at the tip of the plantar side of the big toe. The train-of-four (TOF) watch SX acceleromyograph (MSD BV, Haarlem, The Netherlands) was calibrated prior to the start of the surgery and the TOF counts were determined every $30 \mathrm{~min}$, and whenever an extra rocuronium bolus was requested. At the end of surgery, a TOF count, a TOF ratio, or a post-tetanic count (PTC) was measured at the ulnar nerve (not blinded). If any residual NMB $(\mathrm{TOF}<0.92)$ was present at the ulnar site, sugammadex was given according to the depth of NMB (Table 1). In patients with a PTC of 0 , the potential for recurarization was assessed $30 \mathrm{~min}$ after sugammadex administration, and an additional dose was given if necessary. ${ }^{13}$ In case of a clinical reason to continue mechanical ventilation after surgery, administration of sugammadex was postponed until at least one PTC was measured. 
Table 1 Surgical rating scale (SRS) and depth of neuromuscular block (NMB)

\begin{tabular}{|c|c|c|}
\hline \multicolumn{3}{|l|}{ SRS category* } \\
\hline 1 & \multicolumn{2}{|c|}{$\begin{array}{l}\text { Extremely poor conditions: the surgeon is unable to work because of coughing or because of } \\
\text { laparoscopic field because of inadequate muscle relaxation. Additional neuromuscular blo }\end{array}$} \\
\hline 2 & \multicolumn{2}{|c|}{$\begin{array}{l}\text { Poor conditions: there is a visible laparoscopic field, but the surgeon is severely hampered by } \\
\text { with continuous muscle contractions, movements, or both with the hazard of tissue damag } \\
\text { blocking agents must be given }\end{array}$} \\
\hline 3 & \multicolumn{2}{|c|}{$\begin{array}{l}\text { Acceptable conditions: there is a wide visible laparoscopic field but muscle contractions, move } \\
\text { causing some interference with the surgeon's work. Additional neuromuscular blocking ag } \\
\text { deterioration }\end{array}$} \\
\hline 4 & \multicolumn{2}{|c|}{$\begin{array}{l}\text { Good conditions: there is a wide laparoscopic working field with sporadic muscle contraction } \\
\text { no immediate need for additional neuromuscular blocking agents unless there is the fear }\end{array}$} \\
\hline 5 & \multicolumn{2}{|c|}{$\begin{array}{l}\text { Optimal conditions: there is a wide visible laparoscopic working field without any movemen } \\
\text { need for additional neuromuscular blocking agents }\end{array}$} \\
\hline NMB category & & Sugammadex dose \\
\hline intense & post-tetanic count, 0 twitches & $16 \mathrm{mg} \cdot \mathrm{kg}^{-1 * *}$ \\
\hline deep & train-of-four $=0$, post-tetanic count $\geq 1$ & $4 \mathrm{mg} \cdot \mathrm{kg}^{-1}$ \\
\hline moderate & train-of-four, 1 to 2 twitches & $2 \mathrm{mg} \cdot \mathrm{kg}^{-1}$ \\
\hline shallow or none & train-of-four, 4 twitches, ratio $>0 \%$ & $2 \mathrm{mg} \cdot \mathrm{kg}^{-1}$ \\
\hline
\end{tabular}

SRS was adapted from Martini et al. ${ }^{2}$ Depth of NMB was scored based on the TOF-count at the end of surgery. Sugammadex dose is adjusted based on NMB. *The surgical rating scale (SRS) is an ordinal scale ranging from 1 to 5 ; **After the first ten patients, the sugammadex dose was increased from $4 \mathrm{mg} \cdot \mathrm{kg}^{-1}$ to $16 \mathrm{mg} \cdot \mathrm{kg}^{-1}$ in intense block

$\mathrm{NMB}=$ neuromuscular blockade; $\mathrm{SRS}=$ surgical rating scale; TOF $=$ train-of-four

Surgical and anesthesia conduct

All patients received standardized care preoperatively and postoperatively according to an enhanced recovery after surgery (ERAS) pathway. ${ }^{14,17}$ All procedures were undertaken at our tertiary teaching hospital with a volume of approx. 150-200 esophagectomies per year where two senior surgeons and a surgical fellow are present during the procedure. A group of ten attending anesthesiologists provide care for these procedures. Either a two-stage thoraco-laparoscopic esophagostomy (Ivor Lewis procedure) or a three-stage thoracolaparoscopic esophagostomy (McKeown procedure) was chosen depending on the tumour location. ${ }^{14-18}$ Laparoscopy (in supine position) was performed at a maximum insufflation pressure of $15 \mathrm{mmHg}$. Thoracoscopy (in prone position) was done with a maximum insufflation pressure of $6-8 \mathrm{mmHg}$.

An epidural catheter was placed preoperatively at the T6-7 or T7-8 in most patients. General anesthesia was induced with propofol $\left(2.0-3.0 \mathrm{mg} \cdot \mathrm{kg}^{-1}\right)$ and sufentanil $\left(0.2-0.3 \mu \mathrm{g} \cdot \mathrm{kg}^{-1}\right)$ and $1.0 \mathrm{mg} \cdot \mathrm{kg}^{-1}$ rocuronium for paralysis. Sevoflurane at an age-adjusted minimal alveolar concentration of 1.0 was used for anesthesia maintenance. Ten millilitres of bupivacaine $0.25 \%$ was administered in two divided boluses via the epidural catheter with an infusion of bupivacaine $0.25 \%(0.08-0.1$ $\mathrm{mL} \cdot \mathrm{kg}^{-1} \cdot \mathrm{hr}^{-1}$ ) continuously administered afterwards. In case of a failed epidural, intravenous ketamine (bolus 0.25 $\mathrm{mg} \cdot \mathrm{kg}^{-1}$, maintenance $0.1 \mathrm{mg} \cdot \mathrm{kg}^{-1}$ ) was started combined with intravenous sufentanil continuously $0.5-1.0$ $\mu \mathrm{g} \cdot \mathrm{kg}^{-1} \cdot \mathrm{hr}^{-1}$.

\section{Outcomes}

The primary endpoint was the SRS assessed during the abdominal phase. The secondary endpoints included: 1) SRS during the thoracic phase of the surgery; 2) indication for, and amount of, rocuronium boluses administered; 3) surgical or anesthesia-related intraoperative adverse events; 4) postoperative numerical pain rating (NRS) up to $12 \mathrm{hr}$ postoperatively; 5) depth of NMB at the end of surgery; 6) time until spontaneous breathing; 7) time until extubation after interruption of sedation; 8) surgical events or complications intraoperatively; and 9) ability of surgeons and anesthesiologists to estimate which group the patient was randomized to (at the end of each of the two phases). Exploratory post hoc assessments included intensive care unit (ICU) and hospital stay, need for reintubation, and postoperative complications. Definitions can be found in the Appendix. Data were collected until death or discharge from the hospital.

\section{Statistical analysis}

The sample size calculation was based on a study by Martini et $a l^{2}$ Those authors showed a difference of 0.7 points on the SRS with a standard deviation (SD) of 0.4. 
Fig. 1 CONSORT flow diagram of the DEPTH trial

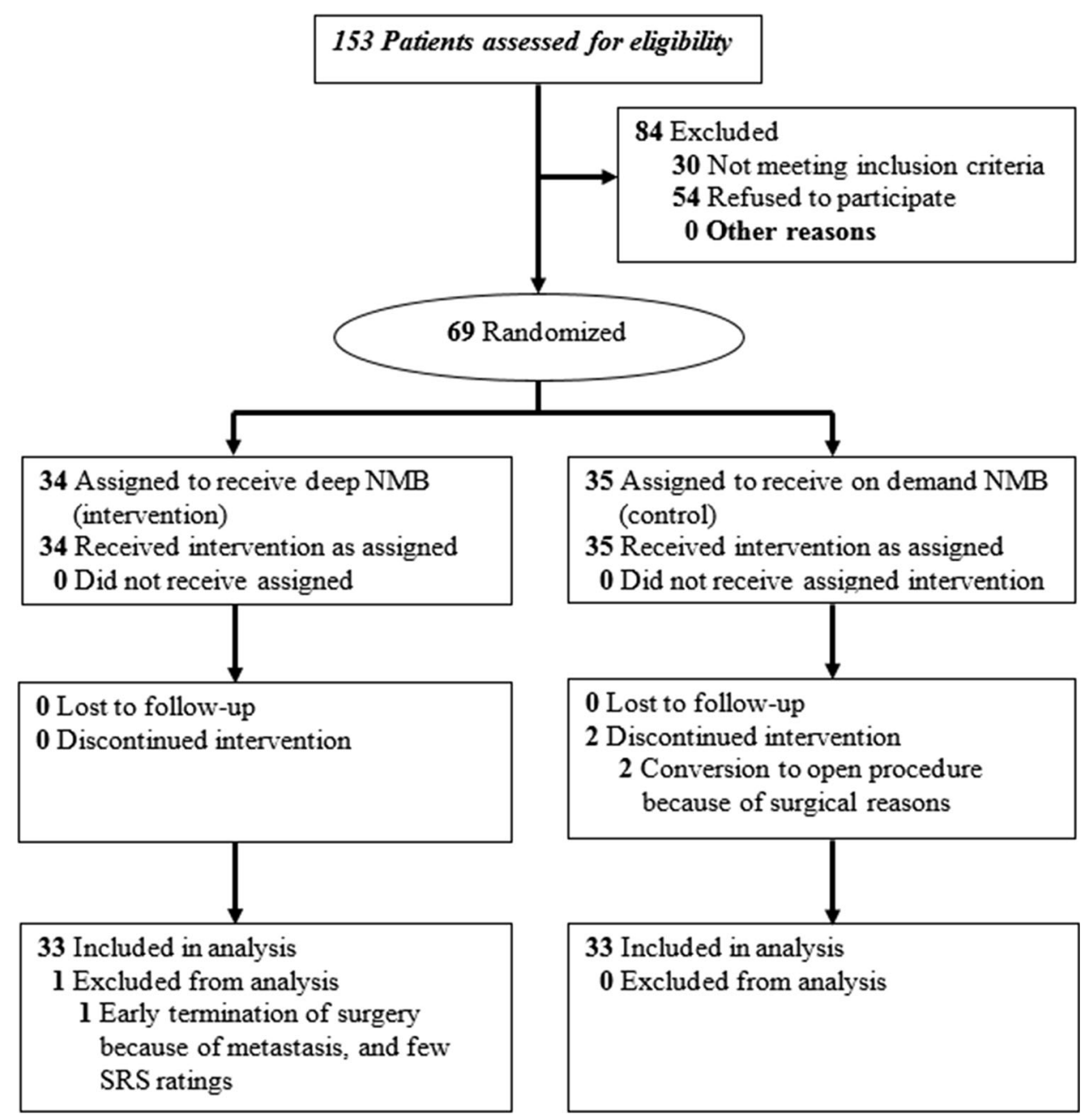

Esophagectomy was anticipated to have more sources of variability for the surgical conditions, which could reduce effect size and increase variability; accordingly, a sample size of 60 was determined to provide a power of $90 \%$ with an alpha of 0.05 in case the effect size was 0.6 points and a SD of 0.7 points on the SRS scale. We included 66 patients for analysis anticipating a $10 \%$ drop-out. Patients who withdrew informed consent, or for whom the study was prematurely stopped because of safety or surgical reasons before any SRS measurements could be made, were replaced and excluded from analysis.

The data analysis was based on an intention-to-treat approach. Continuous variables were expressed by their mean (SD) or median [interquartile range (IQR)]. Categorical variables were expressed as $\mathrm{n}$ and percentage (\%). Our initial protocol specified mean SRS per patient as the primary outcome variable assuming that it is normally distributed. Since we identified post hoc that this assumption is not given, we analysed the median SRS score per patient using Wilcoxon tests. The confidence intervals for the median differences were calculated with the Hodges-Lehmann method. Nevertheless, we also analysed the possible influence of the repeated measures per patient using a linear mixed model. We first focused on the crude effect of the two groups on SRS score. Then the repeated measures were added to the model as a random effect. Both models were compared by analysis of variance. Results are reported in the eTable (available as Electronic Supplementary Material).

Missing NRS pain scores were imputed using multiple imputation (ten imputed data sets) with predictive mean matching. Statistical analysis was performed using $\mathrm{R}$, the statistical package.

\section{Results}

Sixty-nine patients were randomized. Despite the planned intention-to-treat approach, three patients were excluded after randomization because of unforeseen circumstances. 
Table 2 Patient characteristics

\begin{tabular}{lll}
\hline Patient characteristics & $\begin{array}{l}\text { Intervention } \\
(n=33)\end{array}$ & $\begin{array}{l}\text { Control } \\
(n=33)\end{array}$ \\
\hline Male & $28(84 \%)$ & $27(82 \%)$ \\
Age & $61(9 \%)$ & $63(9 \%)$ \\
Weight & $87[64-93]$ & $82[64-96]$ \\
BMI & $26(4)$ & $26(5)$ \\
Cardiovascular disease & $11(33 \%)$ & $15(45 \%)$ \\
COPD/asthma & $5(15 \%)$ & $1(3 \%)$ \\
Diabetes mellitus type 1 or 2 & $2(6 \%)$ & $2(6 \%)$ \\
CVA/stroke & 0 & $2(6 \%)$ \\
Kidney disease & $1(3 \%)$ & $1(3 \%)$ \\
Previous abdominal surgery & $2(6 \%)$ & $3(9 \%)$ \\
Previous thoracic surgery & 0 & $3(9 \%)$ \\
ASA classification & & $6(18 \%)$ \\
I & $8(24 \%)$ & $20(61 \%)$ \\
II & $20(61 \%)$ & $7(21 \%)$ \\
III & $5(15 \%)$ & $5(15 \%)$ \\
Type of resection & & $28(85 \%)$ \\
McKeown & $4(12 \%)$ & $29(88 \%)$ \\
Ivor Lewis & $29(88 \%)$ & $21(64 \%)$ \\
Epidural & & \\
\hline
\end{tabular}

Numbers are in $n(\%)$ and mean (standard deviation) and median [interquartile range]

ASA = American Society of Anesthesiologists; $\mathrm{BMI}=$ body mass index; $\mathrm{COPD}=$ chronic obstructive pulmonary disease; $\mathrm{CVA}=$ cerebrovascular accident

In one patient, the procedure was terminated (because of metastases) before any measures (scoring) were made. The other two patients were withdrawn from the study before start of anesthesia by order of the surgeon because extreme complicated procedures and the need for deep muscle relaxation were expected. The CONSORT diagram is shown in Fig. 1. Table 2 shows the patient characteristics.

Surgical conditions and neuromuscular blockade during surgery

There was no difference between the intervention and control group concerning the median [IQR] SRS scores in the abdominal phase (4 [4-5] vs 4 [4-5], respectively; median difference, $0 ; 95 \% \mathrm{CI}, 0$ to $0 ; P=0.45)$, in the thoracic phase (4 [4-4] vs 4 [4-4], respectively; median difference, $0 ; 95 \% \mathrm{CI}, 0$ to $0 ; P=0.26$ ) and in both phases combined, (4 [4-4] vs 4 [4-4], respectively; median difference, $0 ; 95 \% \mathrm{CI}, 0$ to $0 ; P=0.45$, Table 3 ). When corrected for repeated measures, differences between groups remained non-significant (see Appendix).
The number of SRS observations made every $30 \mathrm{~min}$ was similar between groups. (Table 3). Figure 2 shows that in both groups, SRS was mostly distributed at 4 or higher. In the control group, significantly more on-demand observations of SRS were made than in the intervention group $(P<0.01)$. Surgeons more frequently requested SRS observations than anesthesiologists $(n=121$ vs $65 ; P<$ 0.01 ). Likewise, the amount of on-demand rocuronium boluses requested by both surgeons and anesthesiologists was higher in the control group, $(\mathrm{P}<0.01$, Table 3$)$. Reasons for on-demand bolus requests were field visibility $(34 \%)$, movement or straining $(24 \%)$, high insufflation $(15 \%)$ or respiratory $(9 \%)$ pressures, and others $(17 \%)$.

Figure 3 shows the distribution of TOF scores in both groups over time. In the intervention group, most TOF scores were 0 from the beginning of surgery compared with the control group, in which the scores were highly variable. Figure 4 shows the influence of on-demand rocuronium boluses on associated SRS rating, which increased in both groups $(P<0.01)$.

Neuromuscular blockade reversal and wake up

At the end of surgery, the depth of NMB was significantly different between groups $(P<0.01)$ (Table 3). Five patients in the control group remained with a deep or intense NMB. The intervention group received more sugammadex $(P<0.01)$. Time until complete reversal of NMB, time until termination of administration of the anesthesia, extubation, and the number of patients that needed continued ventilation postoperatively did not differ between groups (Table 3).

Intraoperative events and treatment

The median [IQR] blood loss was similar in control and intervention group patients (200 [123-300] mL vs 200 [100-250] mL, respectively; median difference, 0; 95\% CI, -0 to $99, P=0.14)$. Surgical events occurred in six $(18 \%)$ vs one $(3 \%)$ patient, respectively, $P=0.05$. No intraoperative complications occurred. Only two patients had a cardiac event (rhythm disorder). Pulmonary events defined by a saturation of $<90 \%$ were $18(55 \%)$ vs 15 (45\%), $P=0.46$, respectively. There were no differences in duration of surgery between the two groups $(P=0.95$, Table 3).

\section{Surgeon's and anesthesiologists' group estimation}

When the treatment allocation was the intervention, both surgeons and anesthesiologists more frequently 
Table 3 Intraoperative outcomes

$$
\text { Control }(n=33) \quad \text { Intervention }(n=33) \quad \text { Effect size, odds ratio or }
$$

$P$ value median difference $(95 \% \mathrm{CI})$

\begin{tabular}{|c|c|c|c|c|}
\hline \multicolumn{5}{|l|}{ Primary endpoint } \\
\hline Median SRS during abdominal phase & $4[4-5]$ & $4[4-5]$ & $0(0$ to 0$)$ & 0.45 \\
\hline \multicolumn{5}{|l|}{ Secondary endpoints } \\
\hline Median SRS during thoracic phase & $4[4-4]$ & $4[4-4]$ & $0(0$ to 0$)$ & 0.26 \\
\hline Median SRS both phases & $4[4-4]$ & $4[4-4]$ & $0(0$ to 0$)$ & 0.65 \\
\hline Lowest SRS both phases & $3[2-4]$ & $3[2-4]$ & $0(0$ to 0$)$ & 0.93 \\
\hline All NRS (scale 1-100) & $80[70-90]$ & $80[73-90]$ & $0(0$ to 0$)$ & 0.89 \\
\hline Requested SRS observation, $n / \mathrm{N}(\%)$ & $131 / 512(26 \%)$ & $55 / 434(13 \%)$ & $0.42(0.30$ to 0.59$)$ & $<0.01$ \\
\hline by anaesthesiologists, n/N (\%) & $50 / 512(10 \%)$ & $15 / 434(3 \%)$ & $0.33(0.19$ to 0.58$)$ & $<0.01$ \\
\hline by surgeons, $n / \mathrm{N}(\%)$ & $81 / 512(16 \%)$ & $40 / 434(9 \%)$ & $0.54(0.36$ to 0.80$)$ & $<0.01$ \\
\hline Duration of surgery, min & $426[385-450]$ & $427[395-441]$ & $1(-24$ to 20$)$ & 0.90 \\
\hline On-demand rocuronium, $\mathrm{mg}$ & $81[60-105]$ & $27[0-60]$ & $-57(-87$ to -39$)$ & $<0.01$ \\
\hline Anesthesiologist, mg & $27[0-55]$ & $0[0-23]$ & $-14(-35$ to 0$)$ & $<0.01$ \\
\hline Surgeon, mg & $54[28-78]$ & $25[0-55]$ & $-28(-45$ to -11$)$ & $<0.01$ \\
\hline Bolus requests per patient & $3[3-6]$ & $1[0-2]$ & $-2(-3$ to -1$)$ & $<0.01$ \\
\hline \multicolumn{5}{|l|}{ Group estimation correct abdominal phase* } \\
\hline Anesthesiologist & $17(52 \%)$ & $28(85 \%)$ & $5.27(1.72$ to 16.2$)$ & $<0.01$ \\
\hline Surgeon & $16(48 \%)$ & $27(82 \%)$ & $4.78(1.62$ to 16.1$)$ & $<0.01$ \\
\hline \multicolumn{5}{|l|}{ Group estimation correct thoracic phase* } \\
\hline Anesthesiologist & $24(73 \%)$ & $28(85 \%)$ & $2.10(0.63$ to 7.02$)$ & 0.23 \\
\hline Surgeon & $23(70 \%)$ & $29(88 \%)$ & $3.15(0.91$ to 11.0$)$ & 0.07 \\
\hline \multicolumn{5}{|l|}{ NMB-category at the end of surgery } \\
\hline Intense & $1(0 \%)$ & $27(82 \%)$ & & 0.01 \\
\hline Deep & $4(12 \%)$ & $6(18 \%)$ & & \\
\hline Moderate & $10(30 \%)$ & 0 & & \\
\hline Shallow-none & $18(55 \%)$ & 0 & & \\
\hline \multicolumn{5}{|l|}{ Anesthesia conditions } \\
\hline Sufentanil intravenous, $\mu \mathrm{g}$ & $80[60-125]$ & $110[54-224]$ & $30(-10$ to 62$)$ & 0.24 \\
\hline Sugammadex supplied** & $26(79 \%)$ & $32(97 \%)$ & $8.62(1.33$ to 55.6$)$ & 0.02 \\
\hline Sugammadex dose, $\mathrm{mg}$ & $200[140-240]$ & $650[305-1464]$ & $450(190$ to 1020$)$ & $<0.01$ \\
\hline Time until complete reversal & $3[3-4]$ & $4[3-7]$ & $1(0$ to 2$)$ & 0.26 \\
\hline Time stop anesthesia until extubation, min & $12[5-17]$ & $10[6-17]$ & $2(-4$ to 5$)$ & 0.85 \\
\hline Postoperative ventilation & $6(18 \%)$ & $5(15 \%)$ & $0.80(0.22$ to 2.94$)$ & 0.74 \\
\hline
\end{tabular}

Frequencies are given as $n(\%)$, scaled and ordinal data as median [interquartile range]. The effect sizes of frequencies are presented as odds ratio, those of ordinal or scaled data as median differences together with their $95 \%$ confidence intervals. The surgical rating scale (SRS) is an ordinal scale ranging from 1 to 5 . The SRS were observed every $30 \mathrm{~min}$ and after a bolus of rocuronium requested by the surgeon (on-demand SRS).*Group estimation was evaluated after changing position of the patient and at the end of surgery. **When extubated in the operating room. NRS = numerical pain rating scale

estimated the treatment allocation correctly during the abdominal phase but not during the thoracic phase of the operation (Table 3). Only about $50 \%$ of cases in the control group were estimated correctly. At the end of surgery, assignment to the correct group occurred in $70 \%$ of the cases. Anesthesiologists did not provide better estimations than surgeons $(P=0.71)$.

\section{Postoperative outcomes}

There were no differences in pain scores between groups (Fig. 5). Nor were there any differences in postoperative complications, ICU length of stay, or mortality (Table 4). More patients in the control group (30\% compared with 6\% in the intervention group, $P<0.01)$ had cardiac events afterwards (mostly atrial fibrillation). 


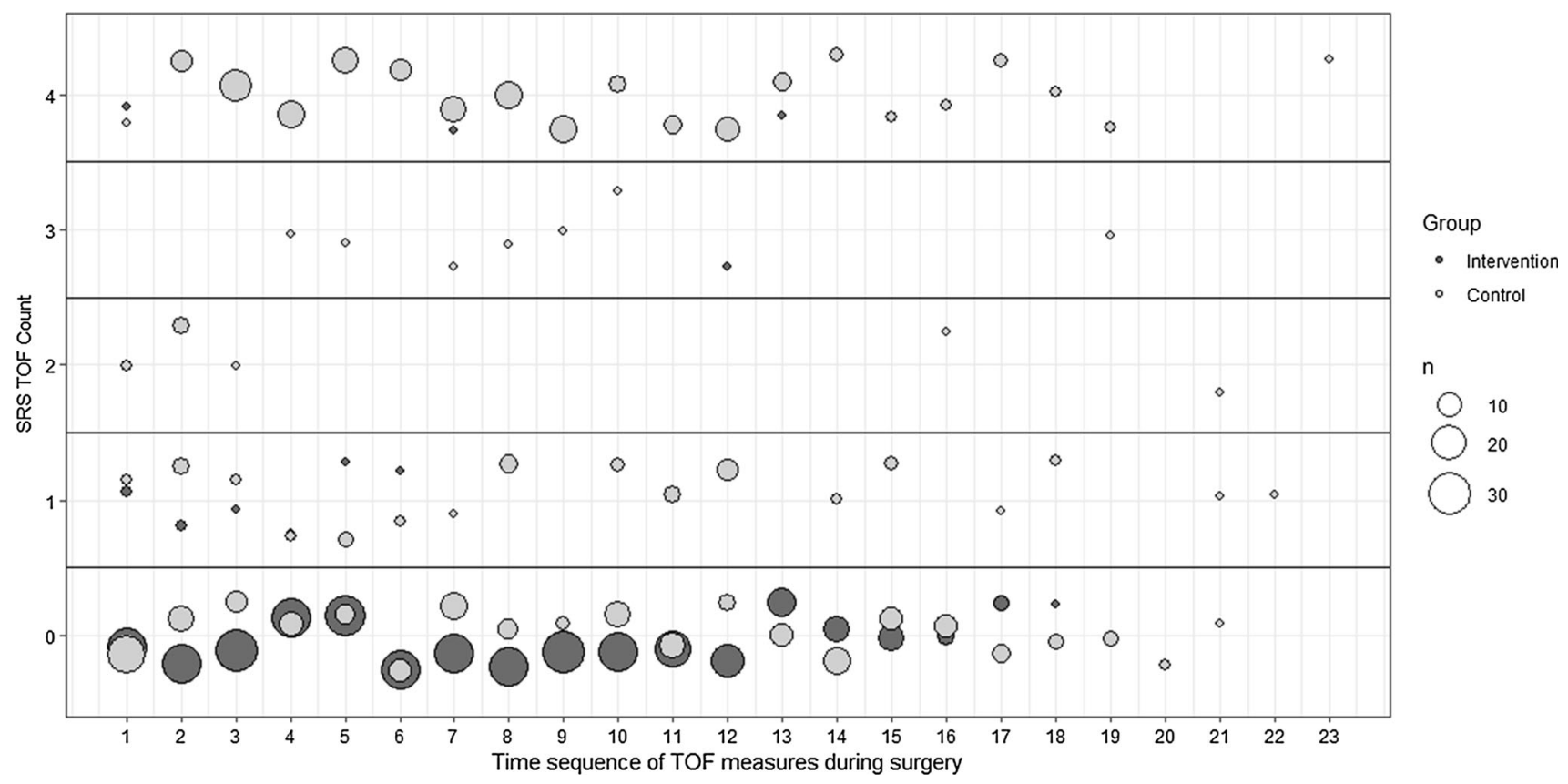

Fig. 2 Train-of-four (TOF) counts of both groups over time. In the intervention group, more patients had a TOF count of zero from the start of the surgery compared with the control group, in which TOF levels varied over time

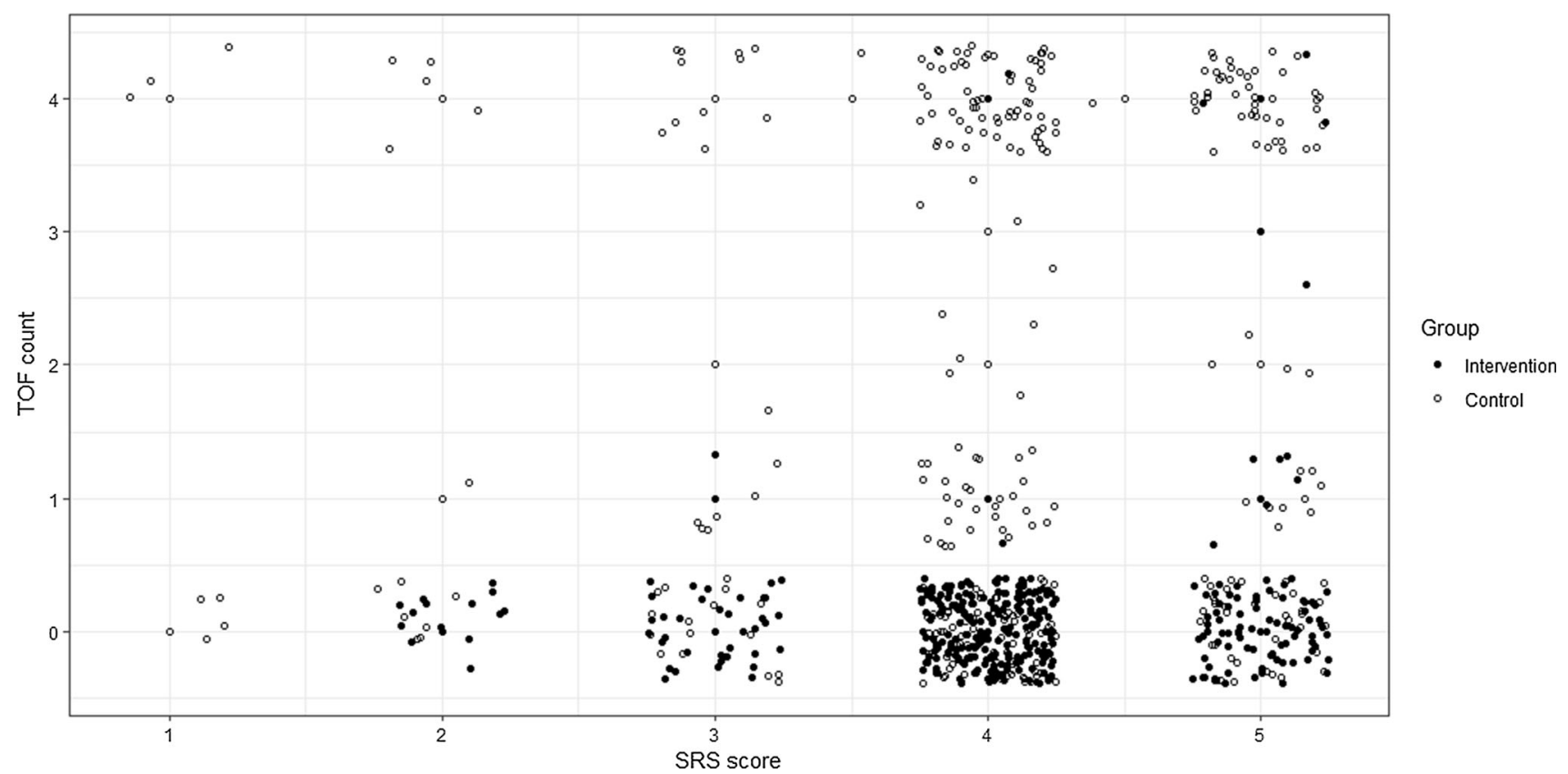

Fig. 3 Relation between train-of-four (TOF) and surgical rating scale (SRS) of all patients (all data points). Most observations in the rocuronium group revealed SRS scores between 4 and 5 at TOF

\section{Discussion}

This randomized-controlled double-blind study in thoracolaparoscopic esophagectomy patients showed that surgical conditions are not improved when aiming at a deep level of counts of 0 . In the control group, few TOF counts were as low as in the intervention group (rocuronium)

NMB by a rocuronium infusion compared with an ondemand only NMB strategy with rocuronium boluses.

Our outcome results are consistent with a recent study by Baete $e t a l .{ }^{8}$ They also reported no improvement in surgical conditions with deep NMB compared with 


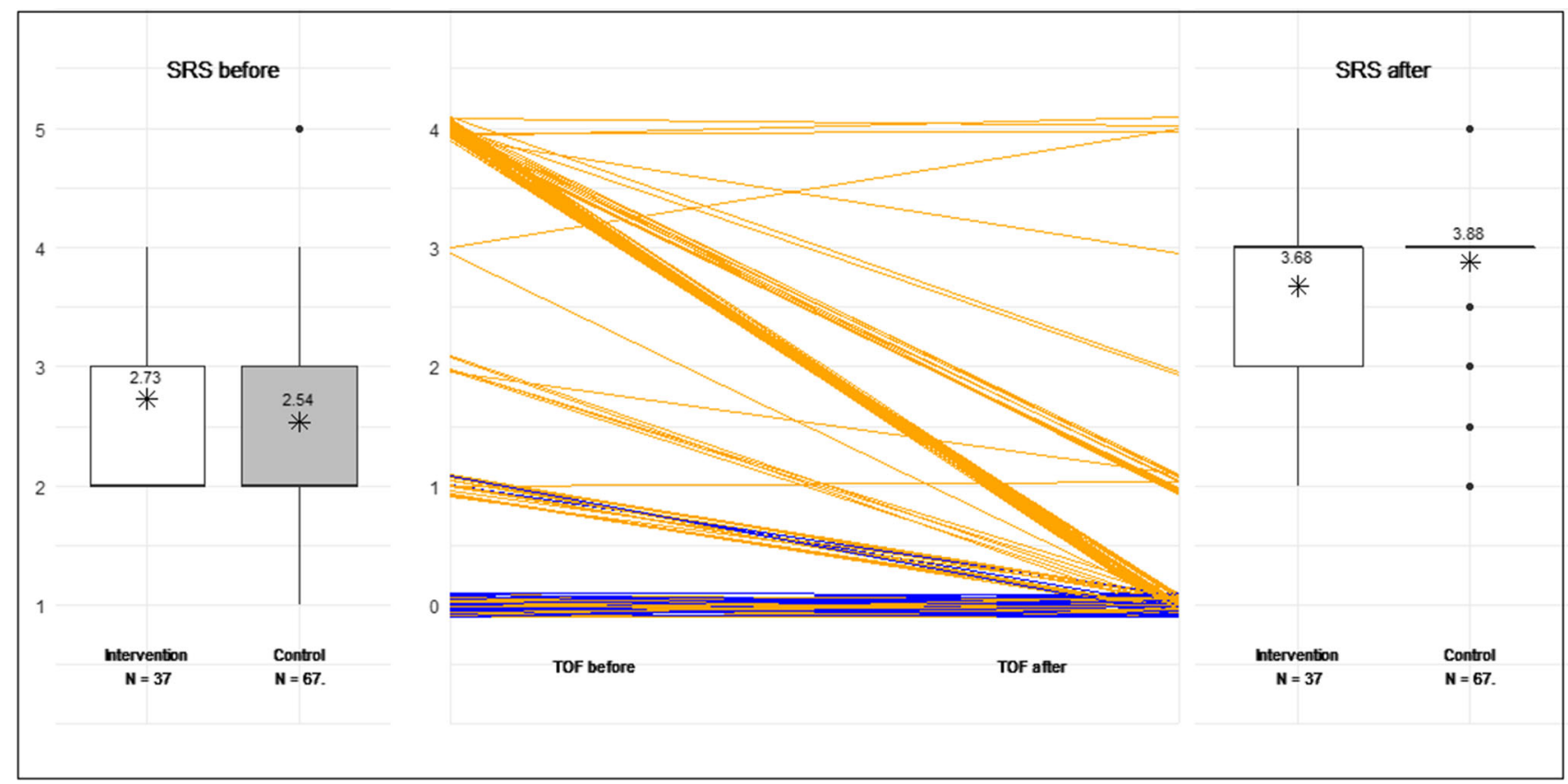

Fig. 4 Box plots of surgical rating scale (SRS) scores before and after on-demand boluses of rocuronium in the intervention group (rocuronium) and control group $(\mathrm{NaCl} 0.9 \%)$ (left and right panel).

moderate NMB in patients undergoing laparoscopic bariatric surgery. Nevertheless, our study contrasts with other studies in patients undergoing laparoscopic surgery where deep NMB was associated with improved surgical conditions and/or improved outcome. , 3,6,7 $^{2}$ Observed SRS scores were generally quite high even when TOF counts were $>0$, suggesting that only relatively few patients needed a deep level of NMB to facilitate surgery. Likewise, we did not find any differences in SRS scores during the thoracic phase. The use of the ribcage and adequacy of lung collapse may have improved surgical conditions.

Earlier studies have compared moderate NMB with deep $\mathrm{NMB}$, mostly leaving it to the discretion of the anesthesiologist to target TOF. ${ }^{5,7,8}$ In this trial, both surgeons and anesthesiologists were blinded to group allocation and neuromuscular monitoring. The number of on-demand boluses requested by surgeons was much higher than those initiated by anesthesiologists. Diaphragmatic contractions can be noticed by surgeons long before anesthesiologists can measure any twitch response to nerve stimulation at any $\operatorname{limb}$ (or before surgical complaints regarding clinical signs of inadequate NMB) ${ }^{19}$ In control patients, surgeons apparently discerned the inferior depth of NMB early enough to avoid impaired surgical conditions. In fact, the higher number of on-
Data are in means $(95 \%$ confidence interval). There was a significantly lower SRS score before an on-demand bolus compared with after $(P<0.01)$. There was no difference between groups

demand boluses of rocuronium in control patients may have led to more acceptable surgical conditions and approximated the SRS score between the treatment groups.

Anesthesiologists and surgeons misjudged group allocation in $30 \%$ of patients, supporting the notion that psychologic factors might have contributed to the request for on-demand boluses. Several factors such as prior surgery, BMI, and analgesic methods have been suggested to influence the need for deep NMB. ${ }^{20-23}$ We cannot verify these relationships with our data, because our study was not stratified for these patient characteristics. Accordingly, it remains unclear which patient objectively requires a deeper NMB for optimal surgical conditions.

We did not find any differences in pain scores in contrast to earlier studies. ${ }^{2,3,6,7}$ Most of the patients received an epidural, which might have masked postoperative pain caused by insufflation. In addition, the use of low abdominal and thoracic pressures may also have contributed to the lack of difference in pain scores. Furthermore, the intervention did not reduce duration of surgery, length of hospital stay, and overall morbidity.

Our study has limitations. The design was chosen to prove the expected differences in surgical conditions. These expected (small) differences, however, were based on those found in an earlier study on a different patient 
Fig. 5 Pain scores until $12 \mathrm{hr}$ postoperatively.

Figures represent mean (standard error) numerical rating scale scores before and after multiple imputation. There were no differences between groups for all time points
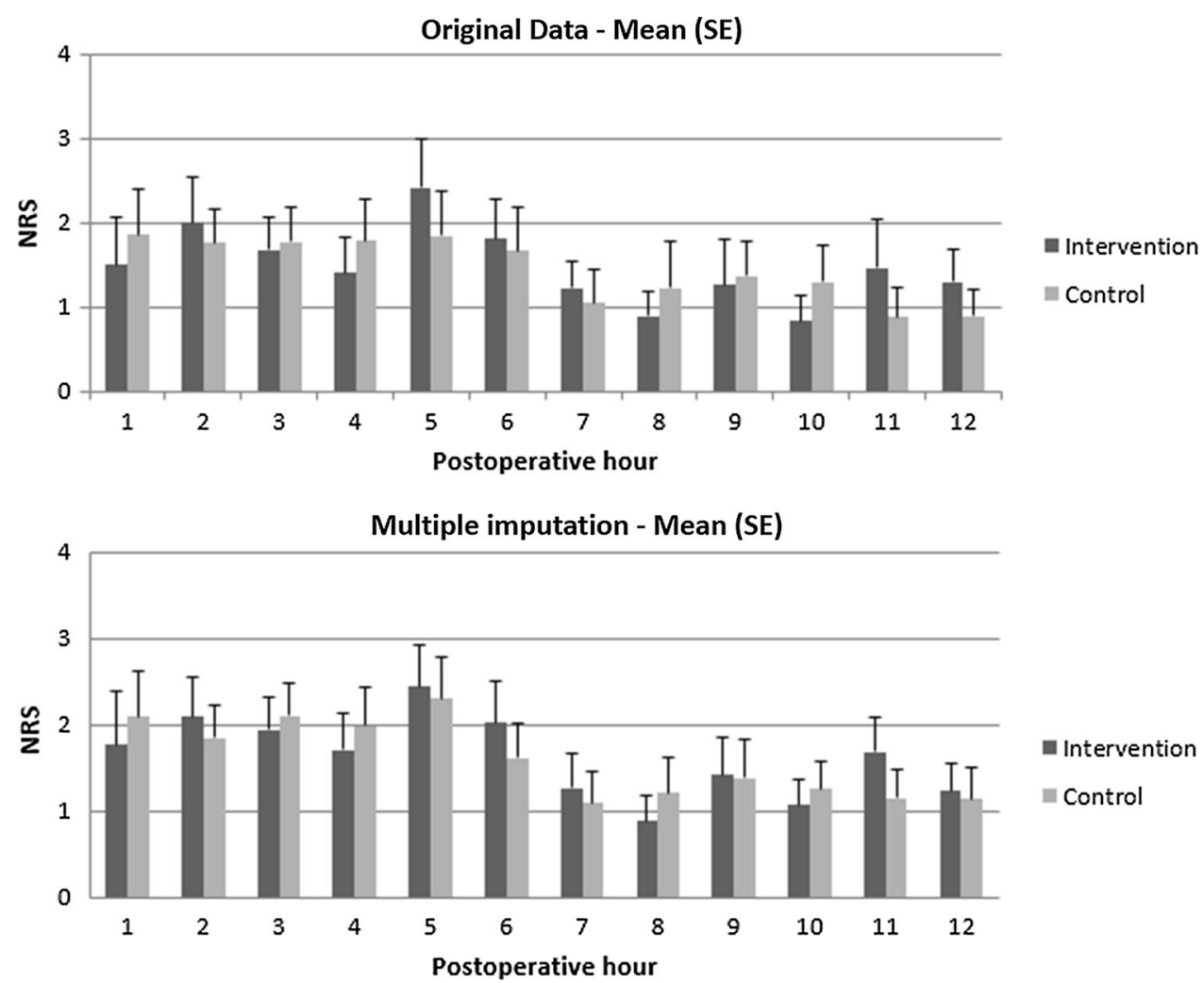

group as no data were available for esophagectomy patients. Furthermore, since SRS scores were not normally distributed, we had to analyze data with nonparametric tests, which usually need a greater sample size than the primarily planned linear models.

Although we included more patients than the sample size calculation recommended, there is insufficient power to conclusively answer the effect of deep NMB on postoperative outcomes and procedural variables. Therefore, these exploratory data do not permit conclusions about the optimal management of NMB on clinically important postoperative outcomes. Nevertheless, the equal rate of postoperative complications suggests that very high numbers of patients are necessary to show advantages of any NMB management approach in patients undergoing thoraco-laparoscopic esophagectomy.

As the dose of sugammadex needed to antagonize an intense NMB block is very high, considerable costs are associated with this regime. Therefore, it was our initial intention to add an economic analysis. Unfortunately, we were unable to proceed with this analysis because of unforeseen difficulties in labelling most costs by the hospital administration.

We measured neuromuscular function at the flexor hallucis brevis muscle following stimulation of the posterior tibial nerve to blind the surgeon as well as the anesthetisiologist. ${ }^{24}$ Since the surgical procedure requires changes between the supine position and prone position with the forearms in front of the head, we performed pilot studies and obtained more stable measurements from the foot under these conditions. Nevertheless, the positioning may have influenced the neuromuscular monitoring. Even more importantly, there is no information available about the correlation between NMB at the flexor hallucis brevis muscle compared with the diaphragm. Therefore, we decided not to adjust the rocuronium infusion rate based on the PTC responses but instead opted for a constant infusion rate. As a result, some patients allocated to deep block ended up with moderate blocks. As we performed the analyses in the intention-to-treat population, these patients' outcomes may have contributed to the negative results of the study.

In conclusion, a constant rocuronium infusion aiming for a deep level of neuromuscular blockade did not improve surgical conditions in a setting that allows for on-demand boluses of rocuronium in all patients. More frequently requested on-demand boluses of rocuronium led to equally acceptable surgical conditions in the respective patients. 
Table 4 Postoperative outcomes

\begin{tabular}{|c|c|c|c|c|}
\hline & Control $(n=33)$ & Intervention $(n=33)$ & $\begin{array}{l}\text { Effect size, odds ratio, or median diff. } \\
(95 \% \mathrm{CI})\end{array}$ & $P$ value \\
\hline Presence of at least one complication & $14(42 \%)$ & $14(42 \%)$ & 1 (1 to1) & 1.00 \\
\hline Pulmonary complications & $11(33 \%)$ & $7(21 \%)$ & $0.54(0.18$ to 1.63$)$ & 0.27 \\
\hline Pneumonia & $5(15 \%)$ & $2(6 \%)$ & & \\
\hline Pneumothorax & $2(6 \%)$ & $1(3 \%)$ & & \\
\hline Lobar collapse & $3(1 \%)$ & 0 & & \\
\hline Pleural fluid & $5(15 \%)$ & $3(9 \%)$ & & \\
\hline Reintubation & $5(15 \%)$ & $2(6 \%)$ & & \\
\hline Cardiac events & $10(30 \%)$ & $2(6 \%)$ & $0.15(0.03$ to 0.64$)$ & 0.01 \\
\hline Surgical complications & $5(15 \%)$ & $8(24 \%)$ & $1.79(0.52$ to 6.20$)$ & 0.36 \\
\hline Post-surgical bleeding & $2(6)$ & 0 & & \\
\hline Mediastinal abscess & $1(3)$ & $1(3)$ & & \\
\hline Abdominal abscess & 0 & 0 & & \\
\hline Anastomotic leakage & $4(12 \%)$ & $4(12 \%)$ & & \\
\hline Chylus (abdominal or thoracic) & $1(3 \%)$ & $4(12 \%)$ & & \\
\hline Gastric tube necrosis & 0 & 0 & & \\
\hline Bowel perforation & 0 & 0 & & \\
\hline Wound infection & 0 & 0 & & \\
\hline Reoperations & $2(6 \%)$ & 0 & & \\
\hline Other & $4(12 \%)$ & $2(6 \%)$ & $0.47(0.08$ to 2.75$)$ & 0.40 \\
\hline Sepsis & $1(3 \%)$ & 0 & & \\
\hline Urologic & $2(6 \%)$ & 0 & & \\
\hline Trombo-embolic event & $2(6 \%)$ & $1(3 \%)$ & & \\
\hline Neurologic events & $1(3 \%)$ & $1(3 \%)$ & & \\
\hline Length of stay ICU/PACU, hr & $17[16-18]$ & $17[17-19]$ & $0(0$ to 0$)$ & 0.46 \\
\hline Length of stay hospital, days & $11[8-20]$ & $9[8-12]$ & $-2(-5$ to 0$)$ & 0.06 \\
\hline Re-admittance ICU & $8(24 \%)$ & $2(6 \%)$ & $0.20(0.04$ to 0.93$)$ & 0.03 \\
\hline Re-admittance hospital & $5(15 \%)$ & $6(18 \%)$ & $1.24(0.34$ to 4.56$)$ & 0.74 \\
\hline 30-day mortality & $2(6 \%)$ & 0 & & \\
\hline
\end{tabular}

Frequencies are given as $n(\%)$, scaled and ordinal data as median [interquartile range]. The effect sizes of frequencies are presented as odds ratio, and those of ordinal or scaled data as median difference together with their $95 \%$ CI each. Statistical comparisons are given conservatively because the study was not powered for these exploratory variables

$\mathrm{CI}=$ confidence interval; $\mathrm{ICU}=$ intensive care unit; $\mathrm{PACU}=$ postanesthesia care unit

Acknowledgement The authors thank Hein Fennema, statistician, for his help in designing the study.

Competing interests Denise P. Veelo: Grants and consultancy work for Edwards Lifesciences, Hemologic, MSD, money paid to institution (current). Bart F. Geerts: Grants and consultancy work for Edwards Lifesciences. Manfred Blobner has received funding from MSD and GE Health to give international lectures and chair CME meetings within the last five years. Markus W. Hollmann: Executive Section Editor Pharmacology with Anesthesia \& Analgesia (ongoing). Eurocept BV, CSL Behring BV, MSD-money paid to institution(current). Suzanne S. Gisbertz, Jan M. Binnekade, Rebekka A. Hannivoort, Jan M. Binnekade, and Mark I. van Berge Henegouwen have no competing interests to declare.

Editorial responsibilty This submission was handled by Dr. Hilary P. Grocott, Editor-in-Chief, Canadian Journal of Anesthesia.
Author contributions Denise P. Veelo and Markus W. Hollmann conceived the study. Denise P. Veelo, Suzanne S. Gisbertz, Manfred Blobner, Mark I. van Berge Henegouwen, and Rebekka A. Hannivoort designed the study protocol. All authors contributed to the writing of this manuscript. This study was executed by Rebekka A. Hannivoort, Johanneke A. Bosman, Suzanne S. Gisbertz, and Mark I. van Berge Henegouwen. Data analysis was performed by Denise P. Veelo, Jan M. Binnekade, and Rebekka A. Hannivoort.

Funding This study was funded by a small project grant from Merck \& Co. (MSD BV), Haarlem, The Netherlands. [grant details: IIS:IH101012].

Open Access This article is distributed under the terms of the Creative Commons Attribution-NonCommercial 4.0 International License (http://creativecommons.org/licenses/by-nc/4.0/), which permits any noncommercial use, distribution, and reproduction in any 
medium, provided you give appropriate credit to the original author(s) and the source, provide a link to the Creative Commons license, and indicate if changes were made.

\section{Appendix}

Definitions and other variables

Total morbidity; the presence of a complication.

Chronic obstructive pulmonary disease; diagnosed according to the GOLD classification. Cardiovascular disease; including hypertensive disease with medication, ischemic cardiac disease, and peripheral vascular disease. Diabetes mellitus; Type 1 or 2 , with need for medication. The composite endpoint "one or more complications"; the percentage of patients that had zero complications compared with those with one or more pulmonary or surgical complications.

Pulmonary complications: Atelectasis; clinically relevant atelectasis (lobar collapse on chest radiograph). Pneumonia; radiologic confirmation of an infiltrate, combined with positive cultures and elevated temperature (above 38.5 degrees Celsius or elevated leucocytes or elevated C-reactive protein. Antibiotics were started preemptive and were later guided by bacterial gram stain and culture. 3) Pneumothorax; collection of air between the visceral and parietal pleural surfaces, requiring drainage. Pleural effusion; collection of fluid between the visceral and parietal pleural surfaces, requiring drainage, and acute respiratory failure (partial pressure of arterial oxygen $<60$ mmHg or oxygen saturation $<90 \%$ while breathing ambient air).

Surgical complications: Intraoperative surgical complications are defined as any complication that has a lasting harmful effect on the patient and is not part of the normal surgical procedure. Surgical events are events that are related to the surgical procedure, such as pneumothorax, and have no lasting effect on the patient. Postoperative surgical bleeding was defined as postoperative blood loss requiring blood transfusion and/ or leading to hemodynamic instability. Mediastinal abscess was scored when an abscess was identified by radiologic imaging or intraoperative visualization and required interventional or antibiotic treatment. Anastomotic leakages were recorded when they were clinically manifest and confirmed by physical examination, radiologic imaging, or intraoperative/endoscopic visualization. Gastric tube necrosis was scored in case of intraoperative/endoscopic confirmation of ischemic changes in the gastric conduit. Chylothorax was recorded when elevated levels of triglycerides in intrathoracic fluid
(> $1 \mathrm{mmol} \cdot \mathrm{L}^{-1}$ [89 $\mathrm{mg}$ per $\left.\mathrm{dL}\right]$ ) were found. Wound infection was defined as a contaminated wound requiring any type of intervention. Sepsis was scored in case of hemodynamic instability of inflammatory/infectious origin.

Other complications: Thrombo-embolic events were recorded when a pulmonary embolus (embolus detected on spiral computed tomography or a ventilation-perfusion mismatch on a lung scintigram) or deep vein thrombosis (detected on duplex ultrasound) was diagnosed. Neurologic events included delirium, (poly)neuropathy, and cerebrovascular events. Cardiac complications were arrhythmia (any change in rhythm on the electrocardiogram, requiring treatment), myocardial infarction (two or three of the following: previous myocardial infarction, electrocardiographic changes suggesting myocardial infarction, or enzyme changes suggesting myocardial infarction), and left ventricular failure (marked pulmonary edema on a chest radiograph). Kidney function disorder; $>50 \%$ elevation of preoperative creatinine.

\section{References}

1. van Wijk RM, Watts RW, Ledowski T, Trochsler M, Moran JL, Arenas $G W$. Deep neuromuscular block reduces intra-abdominal pressure requirements during laparoscopic cholecystectomy: a prospective observational study. Acta Anaesthesiol Scand 2015; 59: 434-40.

2. Martini CH, Boon M, Bevers RF, Aarts LP, Dahan A. Evaluation of surgical conditions during laparoscopic surgery in patients with moderate vs deep neuromuscular block. Br J Anaesth 2014; 112: 498-505.

3. Blobner M, Frick CG, Stäuble RB, et al. Neuromuscular blockade improves surgical conditions (NISCO). Surg Endosc 2015; 29: 627-36.

4. Staehr-Rye AK, Rasmussen LS, Rosenberg J, et al. Surgical space conditions during low-pressure laparoscopic cholecystectomy with deep versus moderate neuromuscular blockade: a randomized clinical study. Anesth Analg 2014; 119: 1084-92.

5. Rosenberg J, Herring WJ, Blobner M, et al. Deep neuromuscular blockade improves laparoscopic surgical conditions: a randomized controlled study. Adv Ther 2017; 34: 925-36.

6. Madsen MV, Staehr-Rye AK, Gätke MR, Claudius $C$. Neuromuscular blockade for optimising surgical conditions during abdominal and gynaecological surgery: a systematic review. Acta Anaesthesiol Scand 2015; 59: 1-16.

7. Torensma B, Martini $\mathrm{CH}$, Boon $\mathrm{M}$, et al. Deep neuromuscular block improves surgical conditions during bariatric surgery and reduces postoperative pain: a randomized double blind controlled trial. PLoS One 2016; 11: e0167907.

8. Baete $S$, Vercruysse $G$, Vander Laenen $M$, et al. The effect of deep versus moderate neuromuscular block on surgical conditions and postoperative respiratory function in bariatric laparoscopic surgery: a randomized, double blind clinical trial. Anesth Analg 2017; 124: 1469-75.

9. Madsen MV, Staehr-Rye AK, Claudius C, Gätke MR. Is deep neuromuscular blockade beneficial in laparoscopic surgery? Yes, probably. Acta Anaesthesiol Scand 2016; 60: 710-6. 
10. Sasaki N, Meyer MJ, Malviya SA, et al. Effects of neostigmine reversal of nondepolarizing neuromuscular blocking agents on postoperative respiratory outcomes: a prospective study. Anesthesiology 2014; 121: 959-68.

11. McLean DJ, Diaz-Gil D, Farhan HN, Ladha KS, Kurth T, Eikermann $M$. Dose-dependent association between intermediateacting neuromuscular-blocking agents and postoperative respiratory complications. Anesthesiology 2015; 122: 1201-13.

12. Groudine $S B$, Soto $R$, Lien $C$, Drover D, Roberts $K$. A randomized, dose-finding, phase II study of the selective relaxant binding drug, sugammadex, capable of safely reversing profound rocuronium-induced neuromuscular block. Anesth Analg 2007; 104: 555-62.

13. Biere SS, van Berge Henegouwen MI, Maas KW, et al. Minimally invasive versus open oesophagectomy for patients with oesophageal cancer: a multicentre, open-label, randomised controlled trial. Lancet 2012; 379: 1887-92.

14. Veelo DP, Gisbertz SS, Hannivoort RA, et al. The effect of ondemand vs deep neuromuscular relaxation on rating of surgical and anaesthesiologic conditions in patients undergoing thoracolaparoscopic esophagectomy (DEPTH trial): study protocol for a randomized controlled trial. Trials 2015; 16: 331 .

15. Health and Youth Care Inspectorate. Rules for clinical trials. Available from URL: https://english.igj.nl/medicines/clinicaltrials-gcp/rules-for-clinical-trials (accessed March 2019).

16. CONSORT Group. Consolidated Standards of Reporting Trials (CONSORT). Available from URL: http://www.consortstatement.org (accessed March 2019).

17. Veelo DP, van Berge Henegouwen MI, Ouwehand $K S$, et al. Effect of goal-directed therapy on outcome after esophageal surgery: a quality improvement study. PLoS One 2017; 12: e0172806.

18. Zhai C, Liu $Y, L i W$, et al. A comparison of short-term outcomes between Ivor-Lewis and McKeown minimally invasive esophagectomy. J Thorac Dis 2015; 7: 2352-8.

19. Donati F, Meistelman C, Plaud B. Vecuronium neuromuscular blockade at the diaphragm, the orbicularis oculi, and adductor pollicis muscles. Anesthesiology 1990; 73: 870-5.

20. Kaafarani HM, Smith TS, Neumayer L, Berger DH, Depalma RG, Itani KM. Trends, outcomes, and predictors of open and conversion to open cholecystectomy in Veterans Health Administration hospitals. Am J Surg 2010; 200: 32-40.

21. Hotouras A, Ribas Y, Zakeri SA, et al. The influence of obesity and body mass index on the outcome of laparoscopic colorectal surgery: a systematic literature review. Colorectal Dis 2016; 18: O337-66.

22. Pikarsky AJ, Saida Y, Yamaguchi T, et al. Is obesity a high-risk factor for laparoscopic colorectal surgery? Surg Endosc 2002; 16: 855-8.

23. Agarwal A, Pandey $R$, Dhiraaj $S$, et al. The effect of epidural bupivacaine on induction and maintenance doses of propofol (evaluated by bispectral index) and maintenance doses of fentanyl and vecuronium. Anesth Analg 2004; 99: 1684-8.

24. Kern SE, Johnson JO, Orr JA, Westenskow DR. Clinical analysis of the flexor hallucis brevis as an alternative site for monitoring neuromuscular block from mivacurium. J Clin Anesth 1997; 9: 383-7.

Publisher's Note Springer Nature remains neutral with regard to jurisdictional claims in published maps and institutional affiliations. 